\title{
Perinatal tolerance to proinsulin is sufficient to prevent autoimmune diabetes
}

\author{
Gaurang Jhala, ${ }^{1,2}$ Jonathan Chee, ${ }^{3}$ Prerak M. Trivedi, ${ }^{1}$ Claudia Selck, ${ }^{1}$ Esteban N. Gurzov, ${ }^{1}$ \\ Kate L. Graham, ${ }^{1,2}$ Helen E. Thomas, ${ }^{1,2}$ Thomas W.H. Kay, ${ }^{1,2}$ and Balasubramanian Krishnamurthy ${ }^{1,2}$ \\ ${ }^{1}$ St. Vincent's Institute, Fitzroy, Victoria, Australia. 'Department of Medicine, St. Vincent's Hospital, The University of \\ Melbourne, Fitzroy, Victoria, Australia. ${ }^{3}$ The Harry Perkins Institute of Medical Research, University of Western Australia, \\ Nedlands, Western Australia, Australia.
}

\begin{abstract}
High-affinity self-reactive thymocytes are purged in the thymus, and residual self-reactive $\mathrm{T}$ cells, which are detectable in healthy subjects, are controlled by peripheral tolerance mechanisms. Breakdown in these mechanisms results in autoimmune disease, but antigen-specific therapy to augment natural mechanisms can prevent this. We aimed to determine when antigen-specific therapy is most effective. Islet autoantigens, proinsulin (PI), and islet-specific glucose-6phosphatase catalytic subunit-related protein (IGRP) were expressed in the antigen-presenting cells (APCs) of autoimmune diabetes-prone nonobese diabetic (NOD) mice in a temporally controlled manner. PI expression from gestation until weaning was sufficient to completely protect NOD mice from diabetes, insulitis, and development of insulin autoantibodies. Insulin-specific T cells were significantly diminished, were naive, and did not express IFN- $\gamma$ when challenged. This longlasting effect from a brief period of treatment suggests that autoreactive $T$ cells are not produced subsequently. We tracked ICRP ${ }_{206-214}$-specific CD8 ${ }^{+} \mathrm{T}$ cells in NOD mice expressing IGRP in APCs. When ICRP was expressed only until weaning, ICRP ${ }_{206-214}$-specific CD8 ${ }^{+} \mathrm{T}$ cells were not detected later in life. Thus, anti-islet autoimmunity is determined during early life, and autoreactive $T$ cells are not generated in later life. Bolstering tolerance to islet antigens in the perinatal period is sufficient to impart lasting protection from diabetes.
\end{abstract}

Authorship note: T.W.H. Kay and B. Krishnamurthy contributed equally to this work.

Conflict of interest: The authors have declared that no conflict of interest exists.

Submitted: December 21, 2015

Accepted: June 6, 2016

Published: July 7, 2016

Reference information:

JCI Insight. 2016;1(10):e86065

doi:10.1172/jci.insight.86065.

\section{Introduction}

The immune system normally functions to recognize and defend against foreign pathogens by utilizing a highly diverse repertoire of specific $\mathrm{T}$ and $\mathrm{B}$ cells. A large number of these cells recognize self-components and must be eliminated or silenced by a process known as immune tolerance. Autoimmune disease results from failure of tolerance mechanisms. While it is normal to have detectable autoreactive $\mathrm{T}$ cells, clinical disease only affects a minority of the population (1). Restoration of the tolerant state is an important goal in the treatment of autoimmune diseases. Antigen-specific therapy is attractive to reestablish tolerance but has not been successful thus far in autoimmune diseases (2).

One of the major hurdles for antigen-specific therapies is that they are often introduced when immunological markers of autoimmunity, such as autoantibodies, are detectable. At this time, naive antigen-specific $\mathrm{T}$ cells have differentiated into antigen-experienced memory cells and the $\mathrm{T}$ cell responses have diversified beyond the initiating antigen (3). It is difficult to induce immune tolerance in these memory cells.

In contrast, there may be a "window of opportunity" during the neonatal period, a time that has several life-long consequences for the immune system. Epidemiological data in humans and mechanistic studies in animal models indicate that priming of the immune system during the neonatal period critically influences host susceptibility to allergic and autoimmune diseases in later life $(4,5)$. In humans and mice (6), the development of the immune system is said to be "layered," as hematopoiesis occurs in distinct waves. The initial wave of $\mathrm{T}$ cells that develops during early life adopts a tolerogenic fate upon encountering antigens (7). During this period, T cells have reduced capacity for acquisition of effector function due to immature antigen presentation (1). Consistent with this, it was recently shown that Aire, which controls expression of self-antigens in the thymus, was critical only until weaning but that it is dispensable beyond weaning age (8). 
Autoreactivity to proinsulin (PI) is central to autoimmune diabetes development in nonobese diabetic (NOD) mice and in humans (9). Anti-islet autoimmunity in NOD mice is preventable by tolerogenic expression of PI throughout life in the antigen-presenting cells (APCs) (10, 11). However, insulin-based trials to prevent or reverse diabetes in humans have failed to demonstrate therapeutic benefit (12). While factors, including delivery of insulin (instead of PI), which does not cover all the antigenic epitopes and uncertainty over the optimal dose and the most efficacious route of administration, may have contributed to lack of clinical efficacy, timing of intervention appears to be a more critical factor. Based on the evidence presented above, we hypothesize that the perinatal period represents the optimal time for intervention for life-long tolerance. Here, we studied the effect of induced ectopic islet autoantigen expression until weaning on the number and phenotype of antigen-specific T cells, pancreatic islet inflammation (insulitis), and autoimmune diabetes development in NOD mice.

\section{Results}

Conditional expression of PI in NOD mice. To test whether antigen-specific intervention limited to the perinatal period is sufficient to protect from autoimmune diabetes, we generated transgenic NOD mice to enable temporal and cell-specific mouse PI expression in the antigen-presenting cells (APCs). Reporter NOD mice expressing PI under the control of the tetracycline-responsive tetO-CMV promoter were bred to previously described driver NOD mice that express the TetR-VP16 tetracycline transactivator protein (tTA) controlled by the IE $\alpha$-MHC-II promoter, referred to herein as NOD-IE $\alpha$-tTA (TA-NOD) mice (8). This enables conditional expression of PI in thymic and peripheral APCs in a doxycycline-dependent (Dox-dependent) manner in the progeny carrying both transgenes. These mice are referred to as tet-inducible PI (TIP) mice (Figure 1A). Analysis of PI expression revealed that transgenic PI was robustly expressed in the thymus and spleen (Figure 1, B and C). After 1 week of Dox treatment, PI expression dropped to baseline levels in the thymus and spleen, as measured by ELISA (Figure 1B), and was undetectable by Western blotting (Figure 1C). Reexpression of PI upon Dox withdrawal was much slower, and recovery was observed after 3 to 4 weeks of Dox removal (Figure 1, B and C). Thus, PI expression in TIP mice was tightly regulated, inducible, and reversible.

PI tolerance in early life is sufficient to prevent diabetes onset. To dissect the influence of timing of PI expression on tolerance, PI was expressed continuously from gestation until weaning and then turned off to test whether a "tolerogenic vaccination" with PI for a short period in early life imparted lasting immune tolerance (Figure 1D). When PI expression was induced until 3 weeks of life (cohort 3), mice were completely protected from insulitis and diabetes, similar to mice that had PI expression induced throughout life (cohort 2) and previously described NOD PI mice (10). Peri-islet infiltrate was observed in a fraction of islets screened from mice in cohort 3 at 12 to 15 weeks of age; however, the insulitis did not progress further when examined at the later age of around 30 weeks. In the control mice with no induction of PI expression (cohort 1), the severity of insulitis and diabetes was similar to WT-NOD mice (Figure 1, E and $\mathrm{F}$ and Supplemental Figure 1A; supplemental material available online with this article; doi:10.1172/jci. insight.86065DS1). When PI expression was limited to gestation and turned off at birth in TIP mice, no protection from diabetes was observed (Supplemental Figure 1B), indicating that PI expression in APCs confined to gestation does not affect diabetes development. Moreover, splenocytes from mice that had PI expression induced until weaning lacked diabetogenic potential, as they were unable to transfer diabetes to irradiated NOD recipients, similar to splenocytes from mice that expressed PI throughout life. In contrast, splenocytes from mice with no induction of PI expression retained their diabetogenic potential (Figure 1G). Temporal tolerance to PI also affected the response to the downstream antigen islet-specific glucose-6-phosphatase catalytic subunit-related protein (IGRP) (9), as the number of IGRP ${ }_{206-214}$-specific $\mathrm{CD}^{+} \mathrm{T}$ cells was significantly reduced in mice that had PI expressed until 3 weeks of life or throughout life (Supplemental Figure 1C). Mice with no induction of PI showed expansion of IGRP 206-214 $_{\text {-specific }}$ $\mathrm{CD}^{+} \mathrm{T}$ cells comparable to WT-NOD mice (Supplemental Figure 1D). As PI-specific CD4 ${ }^{+} \mathrm{T}$ cell help is required for B cell humoral response (13), we measured insulin autoantibodies (IAA). IAA in 12- to 15-week-old prediabetic mice that had PI expressed until 3 weeks of life or throughout life were similar to those of nonautoimmune prone C57BL/ 6 mice, indicating lack of humoral autoimmunity towards insulin in these cohorts (Figure 1H). In contrast, mice with no induction of PI expression had IAA comparable to those of WT-NOD mice (Supplemental Figure 1E), indicating spontaneous anti-insulin autoimmunity. NOD mice express IAA in a heterogeneous manner, with IAA being detectable as early as 4 weeks of age, 
A

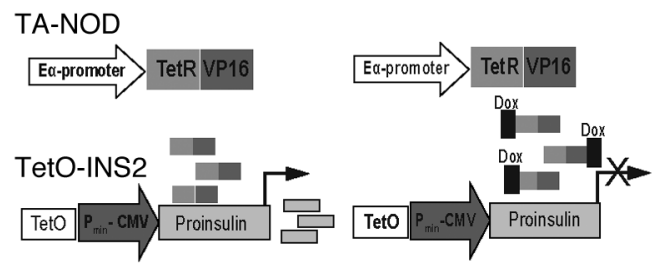

C

Dox $7 \mathrm{~d} \quad$ No Dox WT TIP 14d 21d 30d

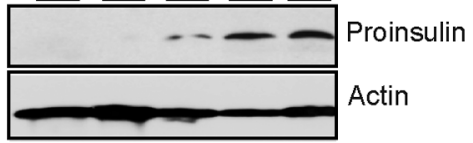

D

\begin{tabular}{l|l|l|l|l|l|}
\hline Cohort 1 & Gestation & Weaning & Insullitis onset & Insulitis & Diabetes \\
\hline
\end{tabular}

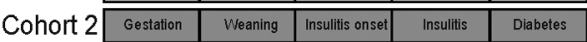

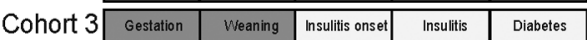

\section{B}

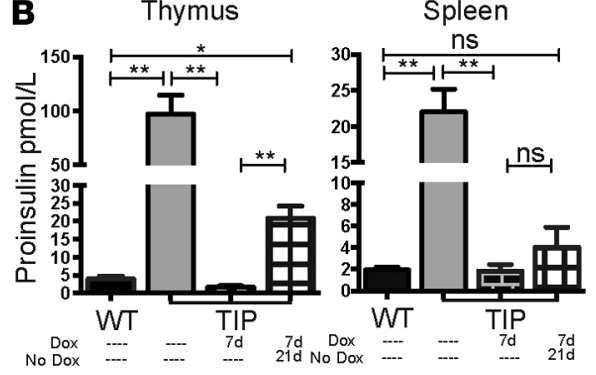

E

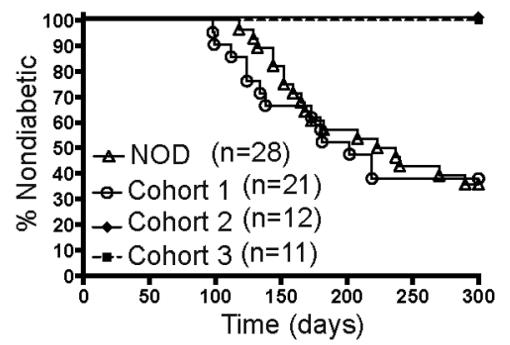

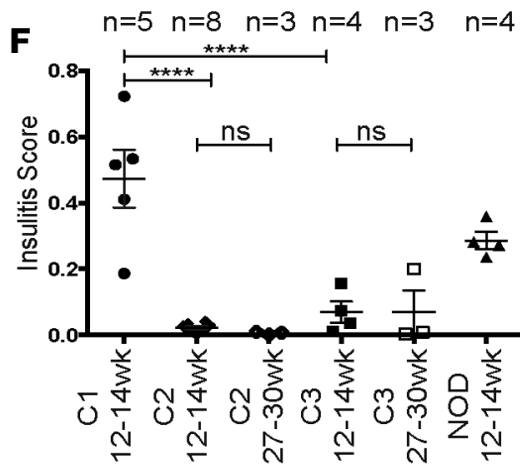
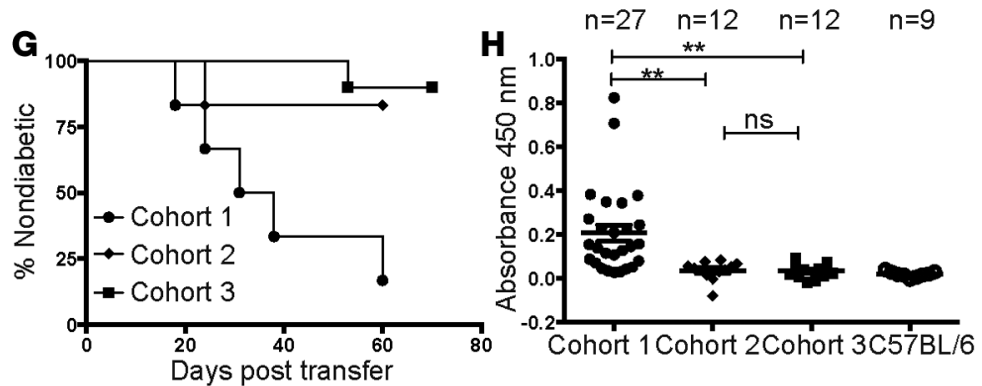

Figure 1. Temporal proinsulin expression and spontaneous diabetes development in TIP mice. (A) Scheme for generation of tetracycline-regulated NODIE $\alpha$-tTA (TA-NOD) and TetO-Ins2 dual-transgenic mice, referred to herein as TIP mice. (B) ELISA assay showing Dox-regulated proinsulin expression (mean \pm SEM) in thymic and splenic homogenates of WT-NOD mice, untreated TIP mice, and TIP mice with the indicated duration of Dox treatment. (C) Western blot analysis of proinsulin expression in thymic lysates of WT-NOD mice and TIP mice with indicated treatments (2-3 independent experiments with $n \geq 2$ per group). (D) Study design depicting different cohorts of TIP mice with the duration of induced PI expression indicated in gray. (E) Incidence of diabetes in cohorts 1-3 of TIP and WT-NOD mice. $P=0.0006$ NOD vs. cohort 2; $P=0.0009$ cohort 1 vs. cohort 2; $P=0.0015$ cohort 3 vs. cohort 1 . (F) Individual insulitis scores (mean \pm SEM) for indicated cohorts of TIP mice ( $n \geq 3 /$ group, $>90$ islets scored per mouse). (C) Incidence of diabetes following transfer of splenocytes from 14- to 16-week-old donor TIP mice (cohorts 1-3) into 10- to 12-week-old irradiated NOD recipients ( $n>5$ each, $P=0.03$ cohort 1 vs. cohort 2; $P=0.0012$ cohort $1 \mathrm{vs}$. cohort 3). (H) IAA in sera of 12- to 15 -week-old TIP mice, represented as absorbance at $450 \mathrm{~nm}$ (mean \pm SEM). Each symbol in scatter plots $\left(\mathbf{F}\right.$ and $\mathbf{H}$ ) represents data from an individual mouse. ${ }^{*} P<0.05,{ }^{* *} P<0.01$, ${ }^{* * *} P<0.0001$. Values compared using One-way ANOVA with Tukey's multiple comparisons test (B, $\mathbf{F}$ and $\mathbf{H})$ ). Survival curves (E and $\mathbf{G})$ compared using log-rank test.

reaching their peak by 8 weeks of age, and declining with age (14). To rule out the possibility that lack of IAA in 12- to 15-week-old TIP mice was due to their early appearance, we measured IAA in 5- to 6-weekold NOD mice and TIP mice (cohorts 2 and 3). Similar to TIP mice at 12 to 15 weeks of age, IAA were not detected at 5 to 6 weeks of age as compared with NOD mice (Supplemental Figure 1F), suggesting absence of immune responses to insulin in TIP mice. These results demonstrate that immune tolerance to PI only for a short period in early life is sufficient to completely prevent autoimmunity and diabetes development in NOD mice.

Insulin-specific T cell tolerance to PI in TIP mice. In the previous studies showing protection from diabetes by transgenic expression of PI in the APCs, T cell tolerance to PI was not demonstrated directly $(10,11)$. Here, we demonstrate tolerance to PI in a number of ways. We enumerated the frequency of insulin $\mathrm{B}_{9-23}$-reactive $\mathrm{CD}^{+}$and insulin $\mathrm{B}_{15-23}$-reactive $\mathrm{CD}^{+} \mathrm{T}$ cells using $\mathrm{I}-\mathrm{A}\left(\mathrm{g}^{7}\right)$ and $\mathrm{H}-2 \mathrm{~K}^{\mathrm{d}}$ tetramers, respectively. Surprisingly, insulin $\mathrm{B}_{9-23}$-specific $\mathrm{CD} 4^{+} \mathrm{T}$ cells binding to insulin $\mathrm{B}_{10-23} / \mathrm{I}-\mathrm{A}\left(\mathrm{g}^{7}\right)$ tetramer $(15)$ were detected in all cohorts of mice. However, there was a significant reduction in the absolute number of 
A

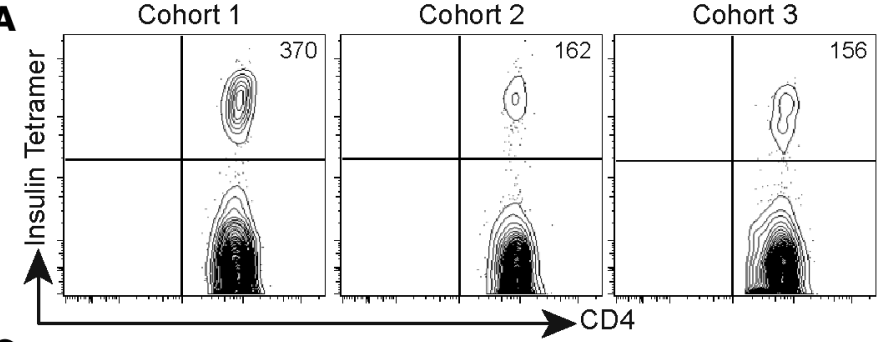

C

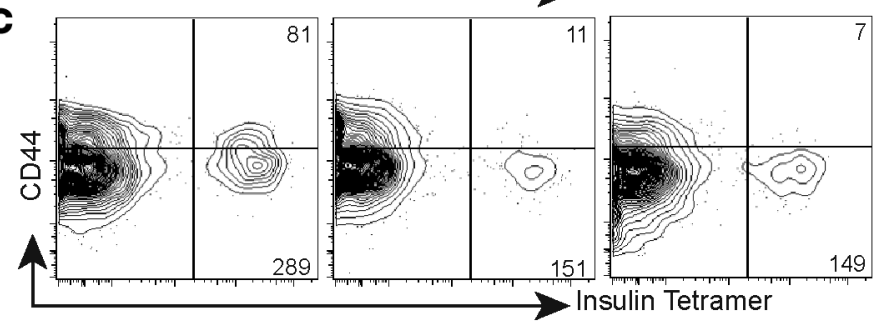

E

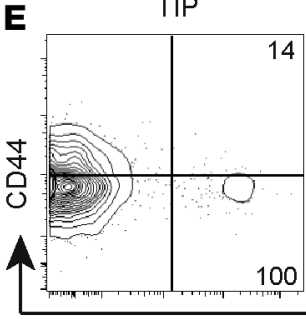

100

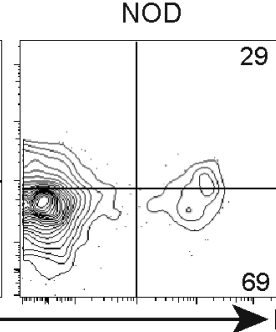

Ins2-KO

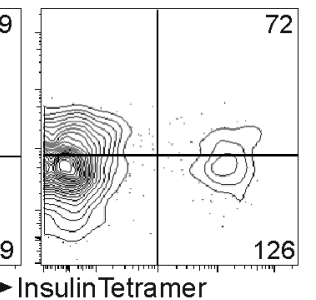

G

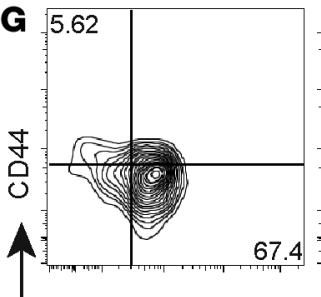

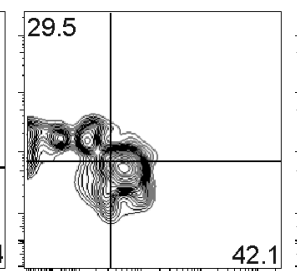

42.1

CD62L
B

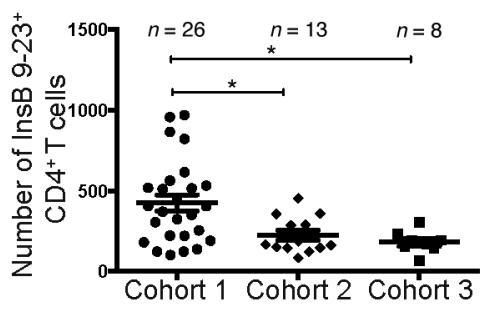

D

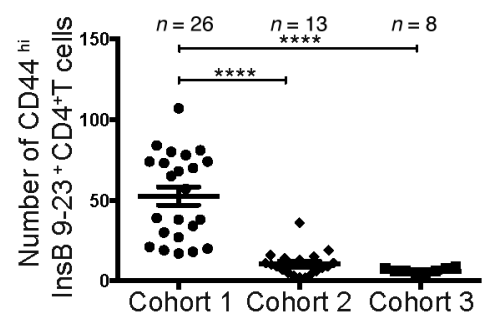

$\mathbf{F}$

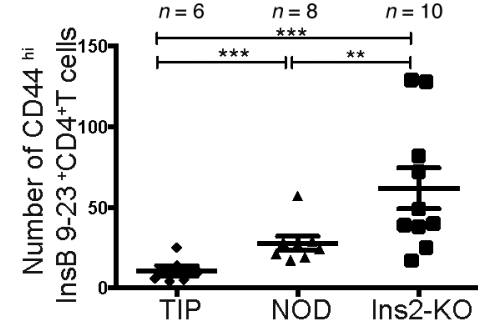

H

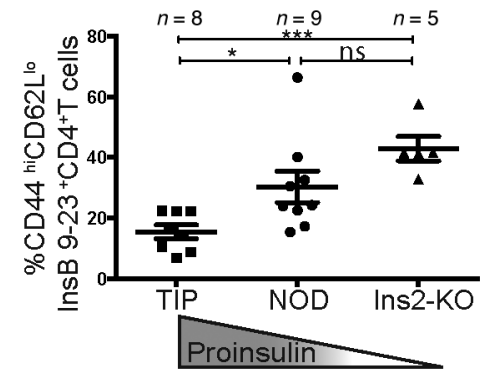

Figure 2. Immune tolerance to antigen-specific T cells in TIP mice. Insulin $B_{10-23}$-specific CD4+ $T$ cells were stained with I- $A$ (g7) tetramer and enriched from pooled peripheral lymphoid organs of 12- to 15-week-old TIP mice (cohorts 1-3), NOD mice, or Ins2.KO mice using magnetic beads and enumerated by flow cytometry. Representative FACS plots ( $A$ and $\mathbf{C}$ ) and enumeration (B and $\mathbf{D}$ ) of insulin $\mathrm{B}_{10-23}$ tetramer ${ }^{+}$CD $4^{+}$T cells and CD44 ${ }^{\text {hi }}$ insulin $B_{10-23}$ tetramer ${ }^{+}$CD4 $^{+}$T cells in TIP mice. Representative FACS plots (E and $\mathbf{G}$ ) and enumeration (F) of insulin $B_{10-23}$ tetramer ${ }^{+}$CD44 ${ }^{\text {hi }}$ CD4 ${ }^{+}$T cells and percent-

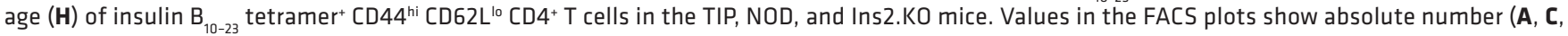
and $\mathbf{E}$ ) and percentage (C) in the indicated gate. Each symbol in the scatter plots (mean \pm SEM) represents data from an individual mouse. ${ }^{*} P<0.05$, ${ }^{* *} P<0.01,{ }^{* *} P<0.001,{ }^{* * *} P<0.0001$. One-way ANOVA with Tukey's multiple comparisons test was used to analyze the data.

insulin $\mathrm{B}_{9-23}$-specific $\mathrm{CD} 4^{+} \mathrm{T}$ cells (Figure 2, $\mathrm{A}$ and $\mathrm{B}$ ). The antigen-experienced $\mathrm{CD} 44^{\text {hi }}$ subset of insulin $\mathrm{B}_{9-23}$-specific $\mathrm{CD} 4^{+} \mathrm{T}$ cells was almost undetectable in mice that had PI expressed until 3 weeks of life or throughout life as compared with mice with no induction of PI expression (Figure 2, C and D). We also investigated the effect of transgenic PI expression in APCs by enumerating $\mathrm{CD} 8^{+} \mathrm{T}$ cells recognizing the insulin $\mathrm{B}_{15-23}$ epitope (16) in TIP mice. Similar to insulin-reactive $\mathrm{CD}^{+} \mathrm{T}$ cells, both the absolute number (Supplemental Figure 2, C and D) and the number of antigen-experienced CD44 ${ }^{\text {hi }}$ (Supplemental Figure 2, $\mathrm{E}$ and $\mathrm{F}$ ) insulin $\mathrm{H}-2 \mathrm{~K}^{\mathrm{d}} \mathrm{B}_{15-23}$ tetramer binding $\mathrm{CD} 8^{+} \mathrm{T}$ cells were significantly reduced in the periphery of TIP mice expressing PI throughout life as compared with mice without any induced expression. This indicates that the remaining insulin $\mathrm{B}_{9-23}$-specific $\mathrm{CD} 4^{+} \mathrm{T}$ cells or insulin $\mathrm{B}_{15-23^{2}}$-reactive $\mathrm{CD} 8^{+} \mathrm{T}$ cells in TIP mice could not become activated by antigen, despite it being expressed widely. The dosage of PI expression in APC had an effect on the frequency of the CD44 hi subset of insulin $\mathrm{B}_{9-23}$-specific $\mathrm{CD} 4^{+} \mathrm{T}$ cells (Figure 2, E and F), as we found that the proportion of the $\mathrm{CD} 44^{\text {hi }} \mathrm{CD} 62 \mathrm{~L}^{\text {lo }}$ (Figure $2, \mathrm{G}$ and $\mathrm{H}$ ) subset of insulin $\mathrm{B}_{9-23}$-specific $\mathrm{CD} 4^{+} \mathrm{T}$ cells is lowest in NOD mice overexpressing PI in APC, intermediate 


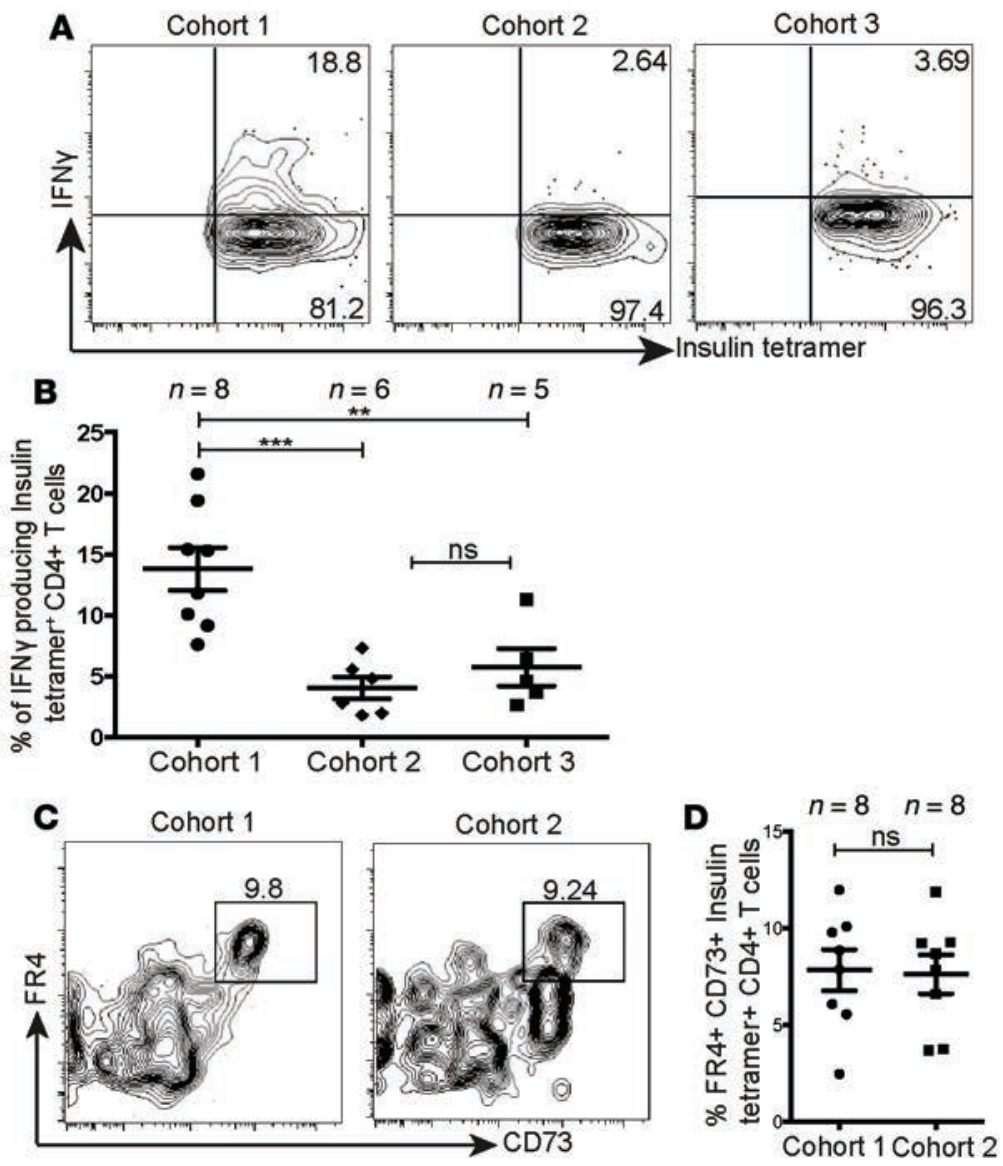

Figure 3. Phenotype of antigen-specific T cells in TIP mice. Insulin $\mathrm{B}_{10-23}$-specific $\mathrm{CD}^{+}{ }^{+} \mathrm{T}$ cells were stained with I-A(g7) tetramer and enriched from pooled peripheral lymphoid organs of 12- to 15-week-old TIP mice in indicated cohorts using magnetic beads and enumerated by flow cytometry. Representative FACS plots (A) and frequency (B) of intracellular IFN- $\gamma$-secreting insulin tetramer ${ }^{+} \mathrm{CD}^{+}{ }^{+} \mathrm{T}$ cells in indicated cohorts of TIP mice. Representative FACS plots (C) and frequency (D) of FR4+ $\mathrm{CD73}^{+}$insulin $\mathrm{B}_{10-23}$ tetramer CD4 ${ }^{+} \mathrm{T}$ cells in TIP mice. Values in the FACS plots show percentage ( $\mathbf{A}$ and $\mathbf{C}$ ) in the indicated gate. Each symbol in the scatter plots (mean $\pm \mathrm{SEM}$ ) represents data from an individual mouse. ${ }^{*} P<0.05$, ${ }^{* *} P<0.01,{ }^{* * *} P<0.001$. Values compared using One-way ANOVA with Tukey's multiple comparisons test (B) and 2-tailed unpaired $t$ test (D).

in NOD mice, and highest in NOD mice with Ins2 gene deleted (17). Collectively, these experiments demonstrate that a few insulin $\mathrm{B}_{9-23}$-specific $\mathrm{CD} 4^{+}$ $\mathrm{T}$ cells and insulin $\mathrm{B}_{15-23}$-specific $\mathrm{CD} 8^{+} \mathrm{T}$ cells (after negative selection of pathogenic $\mathrm{T}$ cells) are detectable in mice with PI expression for the first 3 weeks of life or throughout life.

Residual insulin-specific $T$ cells are functionally impaired. The inability of remaining insulin-reactive $\mathrm{T}$ cells in the periphery of PI-tolerant TIP mice to upregulate activation markers may be due to induction of a state of unresponsiveness or anergy caused by prolonged antigen exposure. To investigate this possibility, we made use of CD73 and FR4, which have been recently described as markers of $\mathrm{T}$ cell anergy $(18,19)$ The frequency of insulin tetramer ${ }^{+} \mathrm{CD} 4^{+} \mathrm{T}$ cells that expressed CD73 and FR4 was similar in TIP mice with and without induction of PI expression (Figure 3, C and D), indicating that induced PI expression did not enhance anergy induction in the residual insulin-reactive $\mathrm{CD} 4^{+} \mathrm{T}$ cells. To further evaluate the functionality of the residual insulin $\mathrm{B}_{9-23}$-specific $\mathrm{CD} 4^{+} \mathrm{T}$ cells, we examined their ability to produce IFN- $\gamma$, as it is a key cytokine implicated in pathogenesis of autoimmune diabetes (20), following stimulation. Following stimulation with PMA and ionomycin, very few insulin $\mathrm{B}_{9-23}$-specific $\mathrm{CD} 4^{+} \mathrm{T}$ cells from mice that had PI expressed until 3 weeks of life or throughout life produced IFN- $\gamma$ as compared with cells from mice with no induction of PI expression (Figure 3, A and B). These data suggest that residual insulin-reactive $\mathrm{CD}^{+} \mathrm{T}$ cells in TIP mice are functionally impaired and that their phenotype in mice expressing PI until 3 weeks is similar to that in mice with life-long PI expression.

Protection from diabetes is not due to antigen-specific Tregs. Antigen expression in thymic APCs has been shown to induce Tregs (21), and Tregs generated during the neonatal period have been shown to be particularly important in protection from autoimmunity (22). To test this, TIP mice were crossed with NOD. FoxP3.GFP reporter mice. No significant difference was observed in the frequency of Foxp $3^{+} \mathrm{GFP}^{+}$insulin $\mathrm{B}_{10-23}$ tetramer ${ }^{+} \mathrm{CD}^{+} \mathrm{T}$ cells in PI-tolerant TIP.Foxp3.GFP mice as compared with WT-NOD.Foxp3.GFP reporter mice (Figure 4, A and B). Furthermore, splenocytes from TIP mice tolerant to PI cotransferred with splenocytes from diabetic NOD mice (1:1 ratio) into NOD.Rag2 ${ }^{-/-}$recipients were unable to delay or prevent diabetes onset in the recipients (Figure 4C), indicating a lack of dominant tolerance in TIP mice. The initial priming of autoreactive $\mathrm{T}$ cells in NOD mice occurs in the draining pancreatic lymph nodes (PLN) as early as 3 weeks of age (23). Therefore, induced PI expression in TIP mice during early life may lead to generation of antigen-specific Tregs in the PLN. We addressed this possibility using two different approaches. First, we enumerated the frequency of insulin tetramer ${ }^{+} \mathrm{Foxp} 3^{+} \mathrm{CD} 4^{+} \mathrm{T}$ cells in PLN of TIP mice in cohort 1 (no PI expression), cohort 2 (continuous PI expression), and cohort 3 (PI expression confined till weaning) (Figure 4D). The absolute number of insulin tetramer binding Foxp $3^{+} \mathrm{CD} 4^{+} \mathrm{T}$ cells was comparable in the PLN of TIP mice in all cohorts (Figure 4E, top left); however, there was a reduction in 

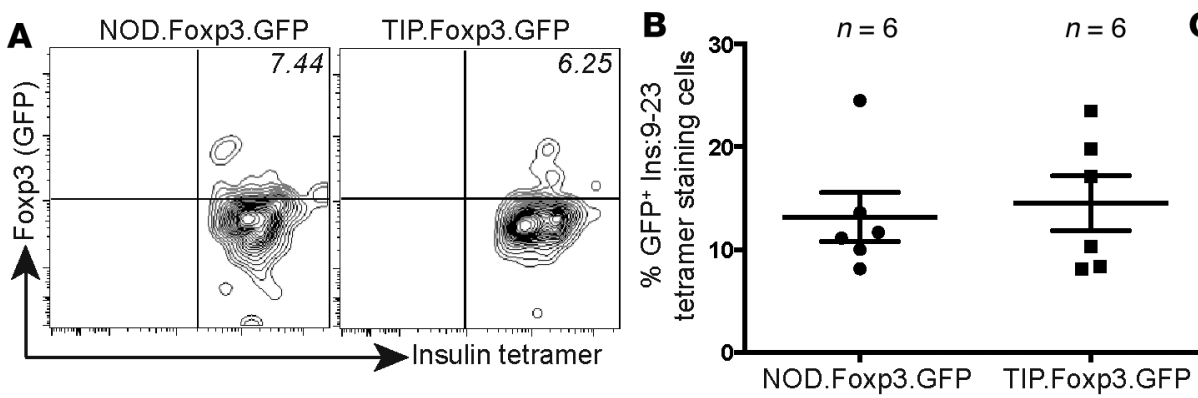

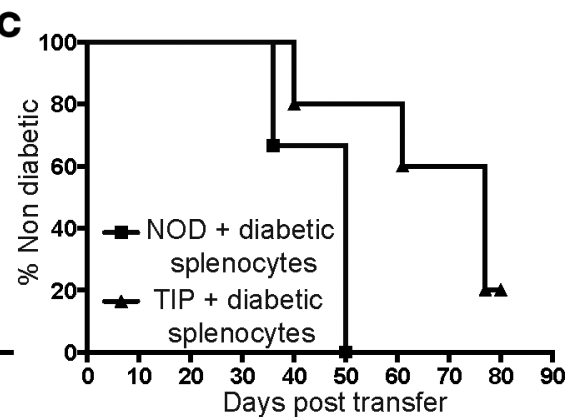

D
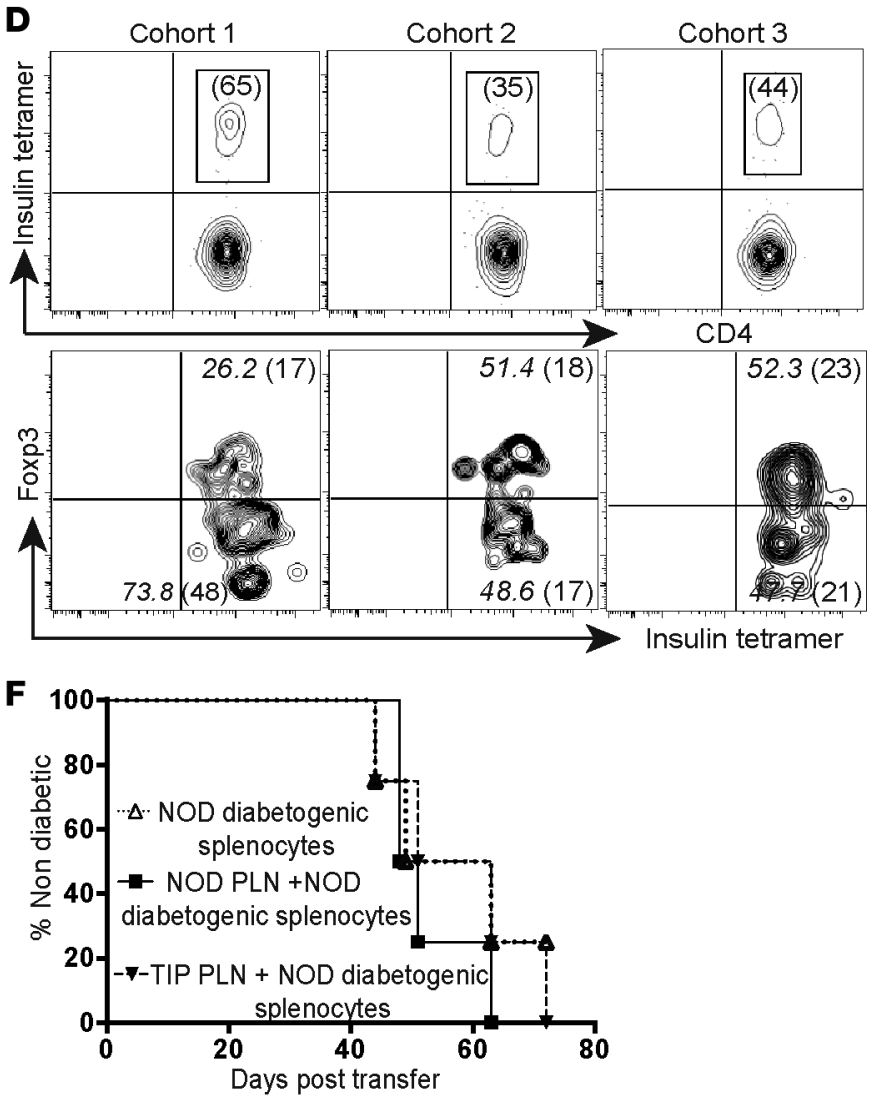
E $\quad$ CD4 $4^{+}$Ins Tet ${ }^{+}$FoxP3 $^{+}$subset

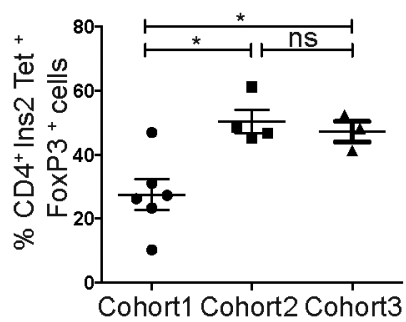

Cohort1 Cohort2 Cohort3

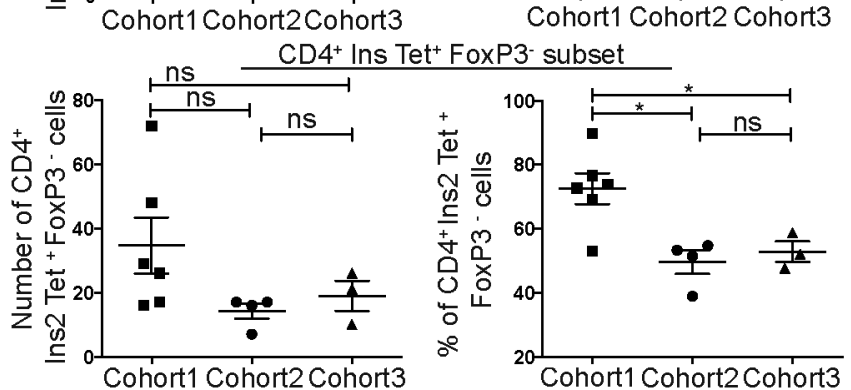

Figure 4. Protection from diabetes in TIP mice is not due to dominant tolerance. TIP mice were bred with NOD.FoxP3.GFP reporter mice. Frequency of Foxp3-expressing insulin-specific CD4 ${ }^{+} T$ cells was determined by enumerating GFP+ insulin tetramer ${ }^{+}$CD4 $4^{+} T$ cells. (A) Representative FACS plots showing GFP reporter expression on insulin tetramer ${ }^{+} \mathrm{CD} 4^{+} \mathrm{T}$ cells and (B) frequency of GFP+ insulin tetramer $\mathrm{CD}^{+} \mathrm{T}$ cells enriched from peripheral lymphoid organs of indicated mice. Each symbol in the scatter plot (mean \pm SEM) represents data from an individual mouse. (C) Incidence of diabetes in 8- to 9-week-old NOD.Rag ${ }^{-/-}$recipient mice after coinjection of splenocytes from diabetic NOD mice along with splenocytes from either nontransgenic NOD mice $(n=3$ recipients) or TIP mice ( $n=5$ recipients) at an equal ratio $\left(2 \times 10^{7}\right.$ cells of each per recipient) $\left(P=\right.$ values were not significant). Insulin ${ }_{10-23}$ tetramer CD4 $^{+} T$ cells were enriched from PLNs of TIP mice, and intracellular FoxP3 expression was analyzed by flow cytometry. Representative FACS plots (D) showing FoxP3 expression on insulin tetramer cells in cohort 1-3 of TIP mice. Cumulative data from 3-4 independent experiments (E) showing number and proportion of CD4+ tetramer+ Foxp3+ Treg subset (top) and CD4+ tetramer+ Foxp3- T effector subset (bottom). Each symbol in the scatter plots (E) represents data from pancreatic lymph nodes (PLNs) pooled from 3 mice (Mean \pm SEM). Values in FACS plots (A and $\mathbf{D})$ shown in italics indicate percentage and values in parentheses indicate absolute numbers. Incidence of diabetes $(\mathbf{F})$ in 8- to 9-week-old NOD.Rag ${ }^{-1-}$ recipient mice after coinjection of splenocytes from diabetic NOD mice alone or along with cells from PLN of 12- to 14-week-old nontransgenic NOD mice or TIP mice (cohort 3 ) at an equal ratio ( $2 \times 10^{7}$ cells of each per recipient, $n=4$ recipients per group, $P=$ values were not significant). ${ }^{*} P<0.05$. Data analysed using 2-tailed unpaired $t$ test (B) or One-way ANOVA with Tukey's multiple comparisons test (E). Survival curves (C and $\mathbf{F})$ compared using log-rank test.

the number of insulin tetramer ${ }^{+}$Foxp $^{-}{ }^{-} \mathrm{CD}^{+}$effector cells in the PLN of TIP mice in cohorts 2 and 3 as compared with cohort 1 (Figure $4 \mathrm{E}$, bottom left). The reduction in the number of insulin tetramer ${ }^{+} \mathrm{CD} 4^{+}$ effectors altered the ratio of insulin-specific Treg/effector T cells in PLN of TIP mice (cohorts 2 and 3), as evident by the increase in the proportion of insulin tetramer ${ }^{+} \mathrm{CD} 4{ }^{+} \mathrm{FoxP} 3^{+}$Tregs (Figure $4 \mathrm{E}$, top right), and induced a concomitant decrease in proportion of insulin-specific $\mathrm{CD}^{+}{ }^{+}$FoxP3 $3^{-}$effector $\mathrm{T}$ cells in $\mathrm{PLN}$ 

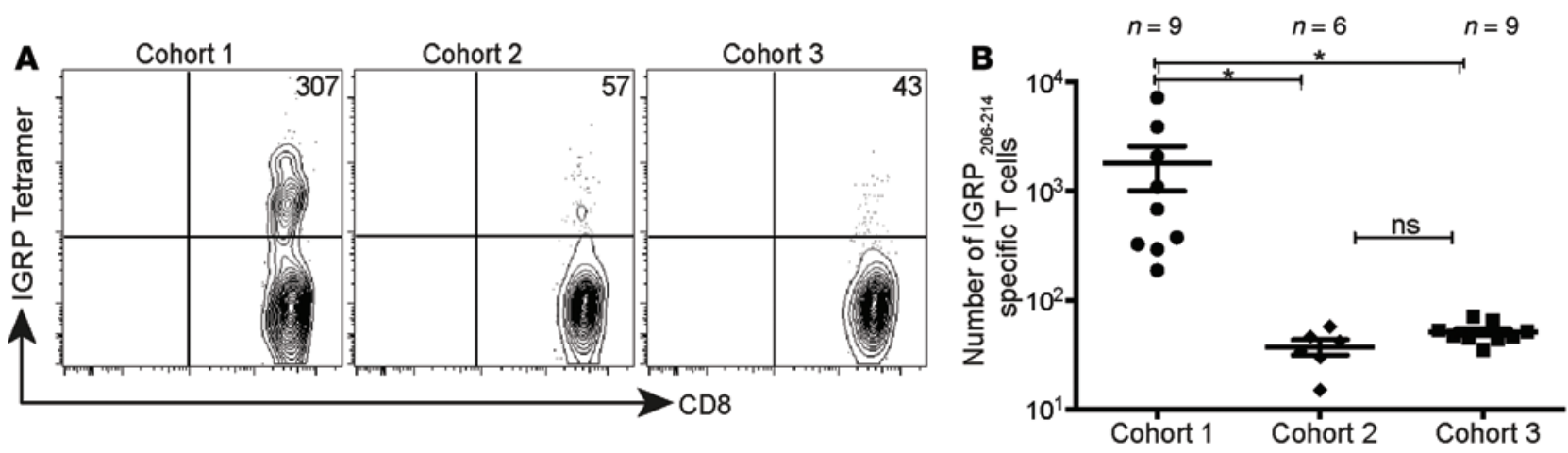

Figure 5. IGRP-reactive $T$ cells develop in early life. (A) Representative FACS plots showing the absolute number of tetramer IGRP $206-214^{-5 p e c i f i c ~ C D 8} 8^{+} T$ cells and (B) enumeration of IGRP $206-214^{-}$-specific CD8 ${ }^{+}$T cells enriched from peripheral lymphoid organs of 12- to 14-week-old TII mice (cohorts 1-3). Values in the FACS plot show absolute number for the indicated gate. Each symbol in the scatter plot (mean \pm SEM) represents data from an individual mouse. ${ }^{*} P<0.05$. Values compared using One-Way ANOVA with Tukey's multiple comparisons test (B).

of TIP mice expressing PI continuously (cohort 2) or until weaning (cohort 3) as compared with those in cohort 1 without induced PI expression (Figure 4E, bottom right). Moreover, the majority of insulin tetramer binding FoxP3 ${ }^{+} \mathrm{CD}^{+}$Tregs detected in PLN were thymus-derived natural Tregs, as determined by their expression of the transcription factor Helios (24) (Supplemental Figure 2G). Second, T cells from PLN of TIP mice with induced PI expression from gestation until weaning were cotransferred at a 1:1 ratio with splenocytes from diabetic WT-NOD mice into NOD.Rag2 $2^{-/-}$recipients to examine their suppressive potential. Transfer of diabetes was similar in all groups tested (Figure 4F). Taken together, these data suggest that protection from diabetes observed in TIP mice expressing PI continuously or until weaning is not due to dominant tolerance exerted by insulin-specific FoxP3 ${ }^{+}$Tregs.

Islet-autoreactive $T$ cells predominantly exit the thymus during the early neonatal period. $\mathrm{IGRP}_{206-214}$-specific $\mathrm{CD}^{+} \mathrm{T}$ cells are the highest frequency antigen-specific T cell in the NOD mouse, and expression of IGRP in APCs completely deletes IGRP ${ }_{206-214}$-specific CD8 ${ }^{+} \mathrm{T}$ cells (9), which makes them suitable to track in vivo after intervention to induce tolerance. To study the mechanism of robust life-long tolerance when autoantigen is expressed only until weaning, we generated tetracycline-inducible IGRP (TII) mice for conditional expression of IGRP in APCs. The conditional expression of IGRP in the APCs of TII mice was validated as previously described (9) (Supplemental Figure 3A). As previously shown (9), transgenic expression of IGRP in TII mice did not effect insulitis progression (Supplemental Figure 3B). We divided these mice into three cohorts similar to TIP mice (Supplemental Figure 3C) and assessed the frequency of IGRP $_{206-214}$-specific $\mathrm{CD}^{+} \mathrm{T}$ cells in the peripheral lymphoid organs of 12- to 15-week-old mice. As previously shown, we found very few cells when IGRP was expressed throughout life, and IGRP ${ }_{206-214}$-specific CD8 $8^{+} \mathrm{T}$ cells could be easily detected in TII mice with no induction of IGRP expression. Interestingly, we also found very few IGRP $_{206-214}$-specific $\mathrm{CD}^{+} \mathrm{T}$ cells when IGRP was expressed until weaning (Figure 5). This indicates that diabetogenic autoreactive $\mathrm{T}$ cells emerge from the thymus only before weaning and that these cells undergo robust tolerance when antigen is expressed in APCs during this period.

\section{Discussion}

We show that early life, before the onset of islet autoimmunity in NOD mice, is a critical period for therapeutic intervention. In children who are genetically at risk for developing type 1 diabetes (T1D), onset of anti-islet autoantibodies peaks between 9 and 24 months of age, with anti-insulin antibody appearing first (25), underscoring the possibility that anti-insulin autoimmunity is established during the first few months of life in subjects with a genetic predisposition to T1D. It has been known for decades that early life is an important time for tolerance induction in immature $\mathrm{T}$ cells, but this study suggests a different but possibly related finding - that autoreactive $\mathrm{T}$ cells are not generated throughout life, as often thought, but are uniquely produced in early life.

Our data both complement and contrast with previous studies from Mathis and Benoist $(8,22)$ that showed that neonatal Aire expression in AIRE-deficient mice, a model of human autoimmune polyglandular syndrome type 1 (APS-1), is sufficient to prevent systemic autoimmunity and enhances the development 
of antigen-specific Tregs. Systemic autoimmunity was prevented on the NOD background, but autoimmune diabetes was restored. While Tregs are an important tolerance mechanism, we did not find evidence that antigen-specific Tregs play a significant role in augmentation of tolerance to PI in our model. APS-1 is a complex and rare disorder involving multiorgan autoimmunity. We have studied the effect of perinatal expression of the single driver antigen PI on development of T1D, a common and prototypical organ-specific autoimmune disease of great clinical significance.

Our findings are consistent with a previous observation from a TCR transgenic mouse model of multiple sclerosis, which suggested that a window of maximum susceptibility to EAE existed at a younger age and that tolerance to myelin-specific $\mathrm{T}$ cells correlated with levels of antigen expression in vivo (26). Moreover, a recent study using a thymus transplant approach demonstrated that thymic production of islet-reactive T cells in NOD mice was limited to a 10-day window after birth (27). Induction of PI expression in APCs in TIP mice during embryonic stages up until the weaning age augments central tolerance to antigenic epitopes of PI presented by thymic APCs by shaping the developing T cell repertoire to be devoid of high-affinity PI-reactive T cells.

Our observations raise the question of whether a tolerance-boosting approach in human T1D patients would mirror the mechanisms observed in NOD mice. The structural and biophysical properties of the MHC class II (I-Ag7) molecule of the NOD mouse and HLA-DQ2-DQ8 are highly conserved, and the MHC peptidome is also identical for both molecules (28). Furthermore, given that the insulin molecule is highly conserved between humans and NOD mice at the amino acid level, it can be envisaged that thymic selection of insulin-reactive $\mathrm{T}$ cells in response to peptide epitopes presented would follow similar principles in both NOD mice and humans.

To translate findings from the animal model presented here into an antigen-specific therapy in the clinic, PI tolerance-boosting therapies will need to be administered to very young children who are at risk of developing T1D. This demands that potential PI-based therapies have a noninvasive mode of delivery. Oral administration of Lactococcus lactis engineered to express PI and IL-10 in combination with anti-CD3 treatment reversed diabetes stably in NOD mice (29); another study administered L. lactis-expressing HSP65 to 4 - to 6-week-old NOD mice and observed decreased incidence of diabetes (30), indicating that mucosal delivery of islet antigens via the gut presents an attractive noninvasive therapy for treatment of T1D. More recently, Mallone and colleagues using a clever approach fused PI to the Fc portion of an antibody to enable the uptake of this fusion protein from mother to fetus via neonatal FcRn receptor. Treatment of pregnant NOD mice late in gestation with PPI.Fc fusion reduced diabetes in the offspring (31). The FcRn receptor is also expressed in the gut in the neonates, thus making a case for "vaccine"-like antigen-specific treatment during perinatal period with a translational potential.

In conclusion, our finding that a brief exposure to PI confined to the perinatal period in NOD mice imparts long-lasting protection from diabetes leads us to suggest that early life is a vulnerable period for the escape of insulin-specific $\mathrm{T}$ cells - a different conclusion to the more accepted view that immature $\mathrm{T}$ cells are susceptible to tolerance induction. Ongoing tolerance therapy to insulin is not required in adult life because few, if any, new autoreactive cells develop then. Of course, some individuals develop T1D very late in life, but this may be from $\mathrm{T}$ cells that originate in early life. Whether this is the case or not, therapeutic interventions targeting PI during early life in high-risk human subjects may prevent diabetes development.

\section{Methods}

Mice. NOD/Lt mice and C57BL/6 mice were purchased from Walter and Eliza Hall animal breeding facility (Kew, Victoria, Australia). NOD-IE $\alpha$-tTA mice were provided by C. Benoist and D. Mathis (Department of Pathology, Harvard Medical School, Boston, Massachusetts, USA). NOD mice expressing GFP under control of FoxP3 promoter (NOD.FoxP3.GFP mice), NOD mice lacking the insulin 2 gene (NOD.Ins2.KO mice), and NOD.RAG1 ${ }^{-/}$mice were obtained from The Jackson Laboratory. NOD8.3 mice express the $\mathrm{TCR} \alpha \beta$ rearrangements of the $\mathrm{H}-2 \mathrm{~K}^{\mathrm{d}}$-restricted, $\beta$ cell-reactive $\mathrm{CD} 8^{+} \mathrm{T}$ cell clone $\mathrm{NY} 8.3$ (32). All mice were bred, maintained, and used under specific pathogen-free conditions at St. Vincent's Institute. All experimental procedures followed the guidelines approved by the institutional animal ethics committee.

Generation of TIP mice. To generate the TetO-Ins2 construct, an 883-bp fragment spanning the coding region of murine proinsulin II (PI) was amplified by PCR from NOD genomic DNA and cloned into BamHI and HindIII sites of the pTRE2 plasmid (Clontech). A 2,868-bp transgene cassette comprising the TetO-minimal CMV promoter, followed by the PI gene and a polyA signal, was excised between 
XhoI and DrdI sites and purified for injection into fertilized NOD/Lt ova using standard techniques. Offspring were screened by PCR using primers spanning the PI gene (5'-GTTGCAGTAGTTCTCCA-3') and the tetO-CMV promoter (5'-GTGAACCGTCAGATCGCCTG-3'). The offspring carrying tetO-Ins2 transgene were crossed with NOD-IE $\alpha$-tTA mice to generate dual-transgenic TIP mice. To turn off PI during gestation and postnatally, pregnant and lactating female mice were fed a diet containing $600 \mathrm{mg}$ Dox/kg food (Specialty Feeds).

Generation of TII mice. To generate the TetO-IGRP construct, a 1,718-bp fragment encompassing IGRP cDNA and polyA signal was excised between SpeI and Cla I restriction sites of the IE-IGRP construct previously described (9) and cloned into NheI (SpeI compatible ends) and ClaI sites of the pTRE2 plasmid (Clontech) under control of the TetO-CMV promoter. The 2,126-bp transgene cassette consisting of the TetO-CMV promoter-IGRP cDNA and polyA signal was excised between Xho I and ClaI sites and purified for injection into fertilized NOD/Lt ova using standard techniques. Offspring were screened by PCR using primers spanning IGRP cDNA (5'-TCAAGATCCCGACTCACG-3') and TRE2 plasmid (5'-GAGATATCGTCGACAAGC-3'). The offspring carrying the TetO-IGRP transgene were crossed with NOD-IE $\alpha$-tTA mice to generate dual-transgenic TII mice.

PI ELISA and Western blotting. Whole thymus and spleen were excised and snap frozen in liquid $\mathrm{N}_{2}$. Frozen tissues were homogenized in ice-cold lysis buffer $(50 \mathrm{mmol} / 1$ Tris- $\mathrm{HCl}, \mathrm{pH} 8,150 \mathrm{mmol} / 1 \mathrm{NaCl}$, $0.5 \%$ Triton X-100) and protease inhibitor cocktail (Sigma-Aldrich) and clarified by centrifugation. PI levels in tissue homogenates were determined using a mouse PI ELISA kit (Mercodia) according to the manufacturer's instructions. Thymic homogenates were resolved by SDS-PAGE and transferred to nitrocellulose membrane using standard procedures. Monoclonal antibodies to insulin (L6B10, Cell Signaling) and $\beta$-actin (Santa Cruz Biotechnology) and horseradish peroxidase-conjugated anti-mouse Ig antibodies were used to detect PI protein.

Diabetes and insulitis. Female mice were monitored for diabetes development for 300 days as described previously (33). Mice with two consecutive blood glucose measurements of greater than or equal to 15 $\mathrm{mM} / 1$ were considered diabetic. Immunohistochemical staining of frozen pancreata for insulitis scoring was performed as previously described (34). For adoptive transfer of diabetes, $2 \times 10^{7}$ splenocytes from TIP mice in cohorts 1-3 were transferred (i.v.) into irradiated (9 Gy) 10- to 12-week-old NOD or NOD.Rag ${ }^{-1}$ recipients, and diabetes development was monitored as above.

Flow cytometry. Antibodies used were anti-CD4 (RM4-5) conjugated to PerCpCy5.5, anti-CD3 (1452C11) conjugated to FITC or anti-CD3 (500 A2) V500, anti-CD44 (1M7) conjugated to AlexaFluor700, anti-CD8a (53-6.7) conjugated to PE-Cy7, anti-CD62L (MEL-14) conjugated to APC-Cy7, and anti-IFN- $\gamma$ (XMG1.2) conjugated to FITC (all BD Biosciences); anti-CD11c (N418), anti-B220 (RA3-6B2), antiCD11b (M1/70), and anti-F4/80 (BM8) conjugated to AlexaFluor450 (all BioLegend); anti-CD8a (5H10) conjugated to Pacific Orange (Invitrogen); and anti-CD73 (ebioTY11.8) conjugated to PE-Cy7, anti-FR4 (ebio12A5) conjugated to FITC, anti-FoxP3 (FJK-16S) conjugated to APC, and anti-Helios (22F6) conjugated to FITC (all eBiosciences). Intracellular staining for IFN- $\gamma$ was performed using the Cytofix/Cytoperm kit (BD Biosciences), and FoxP3 and Helios were stained intracellularly using the FoxP3/Transcription Fixation/Permeabilization kit (eBiosciences). Data were collected on a LSR Fortessa flow-cytometer (BD) and analyzed using FlowJo (Treestar) software.

Tetramer and magnetic bead-based enrichment. The tetramer and magnetic bead-based enrichment method was previously described (35). I-Ag7 tetramers were obtained from $\mathrm{NIH}$ tetramer core, and $\mathrm{H}-2 \mathrm{~K}^{\mathrm{d}}$ tetramers were purchased from ImmunoID. To enrich insulin-specific CD $4^{+} \mathrm{T}$ cells or insulin-specific CD ${ }^{+}$ $\mathrm{T}$ cells, single-cell suspensions $\left(10^{7}\right.$ cells) from peripheral lymphoid organs (pooled spleens and nondraining lymph nodes) or pooled $(3 \times)$ draining PLN were stained with phycoerythrin-conjugated (PE-conjugated) I-Ag7-INS ${ }_{\mathrm{B} 10-23}$ (HLVERLYLVCGGEG) tetramer for 1 hour at room temperature or PE-conjugated $\mathrm{H}-2 \mathrm{~K}^{\mathrm{d}}-\mathrm{INS}_{\mathrm{B} 15-23}$ (LYLVCGGEG) for 1 hour on ice. Hen egg lysozyme I-Ag7-HEL (AMKRHGLDNYRGYSL) tetramer or H-2K $\mathrm{K}^{\mathrm{d}}$-TUM (KYQAVYTTTL) were used as controls. Cells were then washed and stained with anti-PE microbeads (Miltenyi Biotec) followed by magnetic separation using an AutoMACSpro (Miltenyi Biotec) according to the manufacturer's instructions. The separated fractions were stained and analyzed by flow cytometry. $\mathrm{IGRP}_{206-214}$-specific $\mathrm{CD}^{+} \mathrm{T}$ cells $\left(\mathrm{H} 2-\mathrm{K}^{\mathrm{d}}\right.$, VYLKTNVFL) were stained and enriched as previously described (3). Gating strategy for tetramer enrichment was as follows: single cells were gated on forward and side scatter, and dead cells were excluded using propidium iodide. From the live cell population, $\mathrm{CD} 11 \mathrm{c}^{-} \mathrm{CD} 11 \mathrm{~b}^{-} \mathrm{B} 220^{-} \mathrm{F} 4 / 80^{-} \mathrm{CD} 3^{+}$cells were gated as the $\mathrm{T}$ cell population for 
analysis. Further selection of $\mathrm{CD}^{+} \mathrm{T}$ cells or $\mathrm{CD}^{+} \mathrm{T}$ cells was followed by analysis of the insulin or IGRP tetramer ${ }^{+}$population, respectively.

IAA assay. A noncompetitive IAA assay was performed in a 96-well ELISA format as previously described (36). Briefly, an ELISA plate (Costar) was coated with or without human insulin (10 $\mu \mathrm{g} / \mathrm{ml}$, Actrapid, Novo Nordisk) overnight at $4^{\circ} \mathrm{C}$. Wells were blocked with PBS containing $\%$ BSA for 2 hours at room temperature and then probed with sera from 12- to 15-week-old TIP mice, NOD, or C57BL/6 mice (1:10 dilution) for 2 hours. Wells were washed 4 times and a biotinylated anti-mouse IgG1 (AbCam, 1:10,000 dilution) antibody was added for 30 minutes. After washing, horseradish peroxidase-conjugated streptavidin (BioLegend) was added for 15 minutes. The plate was washed 5 times, TMB substrate solution (BioLegend) was added, and absorbance was measured at $450 \mathrm{~nm}$ using a Polarstar (BMG Labtech) microplate reader. Each sample was run in duplicate, and absorbance $(450 \mathrm{~nm})$ of test sample without plate-bound insulin was subtracted from absorbance of test sample with plate-bound insulin to calculate the actual absorbance value for each sample.

CFSE labeling and adoptive transfer. CFSE labeling of $\mathrm{CD}^{+} \mathrm{T}$ cells from NOD 8.3 mice was done as previously described (2). $5 \times 10^{6} \mathrm{CFSE}$-labeled cells were i.v. transferred into TII mice with induced IGRP expression. Hosts were sacrificed after 3 days, and their inguinal and PLN were examined for $\mathrm{CFSE}^{+}$cells.

Immunization and cytokine staining. Insulin $\mathrm{B}_{9-23}(100 \mu \mathrm{g})$ peptide was emulsified in CFA. TIP mice (cohorts 1-3) were immunized s.c. with either CFA alone or CFA plus insulin $\mathrm{B}_{9-23}$ peptide. Insulin tetramer-binding $\mathrm{CD}^{+} \mathrm{T}$ cells were enriched from peripheral lymphoid tissue of TIP mice 12-14 days after priming and stimulated in vitro in complete RPMI medium containing $50 \mathrm{ng} / \mathrm{ml} \mathrm{PMA}$ and $250 \mathrm{ng} / \mathrm{ml}$ ionomycin. Brefeldin A was added 1 hour later, and cells were further stimulated for 3 hours, followed by staining for surface markers and intracellular cytokine staining as per the manufacturer's instructions (BD Biosciences).

Statistics. Groups were compared using 1-way ANOVA with Tukey's multiple comparison test or 2-tailed unpaired $t$ test, as indicated in the figure legends. Diabetes incidence curves were compared using log-rank analysis. Statistical significance was defined as $P$ values of less than 0.05 . Statistical analysis was performed using GraphPad Prism software (version 6.0).

Study approval. All experimental procedures were approved by the institutional ethics committee of St. Vincent's Health and followed the accepted standards of humane animal care.

\section{Author contributions}

GJ performed experiments, analyzed the data, and wrote the manuscript. JC, PMT, CS, ENG, and KLG performed experiments. BK, HET, and TWHK designed the study, analyzed the data, and wrote the manuscript. BK and TWHK supervised the study.

\section{Acknowledgments}

The authors thank S. Fynch, L. Elkerbout, E. Tanuwidjaya, H. Abidin, and R. Boyd (St. Vincent's Institute) for excellent technical assistance and animal husbandry. This work was supported by grants and fellowships from the National Health and Medical Research Council of Australia, the Juvenile Diabetes Research Foundation, and the Operational Infrastructure Support Scheme of the Government of Victoria.

Address correspondence to: Thomas W.H. Kay, St. Vincent's Institute, 41 Victoria Parade, Fitzroy, Victoria 3065, Australia. Phone: 61.3.9288.2480; E-mail: tkay@svi.edu.au.

1. Heninger AK, et al. Activation of islet autoreactive naïve T cells in infants is influenced by homeostatic mechanisms and antigen-presenting capacity. Diabetes. 2013;62(6):2059-2066.

2. Larsson HE, Lernmark A. Vaccination against type 1 diabetes. J Intern Med. 2011;269(6):626-635.

3. Chee J, et al. Effector-memory T cells develop in islets and report islet pathology in type 1 diabetes. J Immunol. 2014;192(2):572580 .

4. Olszak T, et al. Microbial exposure during early life has persistent effects on natural killer T cell function. Science. 2012;336(6080):489-493.

5. Russell SL, et al. Early life antibiotic-driven changes in microbiota enhance susceptibility to allergic asthma. EMBO Rep 2012;13(5):440-447

6. Herzenberg LA, Herzenberg LA. Toward a layered immune system. Cell. 1989;59(6):953-954.

7. Mold JE, et al. Fetal and adult hematopoietic stem cells give rise to distinct T cell lineages in humans. Science. 
2010;330(6011):1695-1699.

8. Guerau-de-Arellano M, Martinic M, Benoist C, Mathis D. Neonatal tolerance revisited: a perinatal window for Aire control of autoimmunity. J Exp Med. 2009;206(6):1245-1252.

9. Krishnamurthy B, et al. Responses against islet antigens in NOD mice are prevented by tolerance to proinsulin but not IGRP. $J$ Clin Invest. 2006;116(12):3258-3265.

10. French MB, et al. Transgenic expression of mouse proinsulin II prevents diabetes in nonobese diabetic mice. Diabetes. 1997;46(1):34-39.

11. Jaeckel E, Lipes MA, von Boehmer H. Recessive tolerance to preproinsulin 2 reduces but does not abolish type 1 diabetes. Nat Immunol. 2004;5(10):1028-1035.

12. Staeva TP, Chatenoud L, Insel R, Atkinson MA. Recent lessons learned from prevention and recent-onset type 1 diabetes immunotherapy trials. Diabetes. 2013;62(1):9-17.

13. Krishnamurthy B, et al. Autoimmunity to both proinsulin and IGRP is required for diabetes in nonobese diabetic 8.3 TCR transgenic mice. J Immunol. 2008;180(7):4458-4464.

14. Yu L, et al. Early expression of antiinsulin autoantibodies of humans and the NOD mouse: evidence for early determination of subsequent diabetes. Proc Natl Acad Sci USA. 2000;97(4):1701-1706.

15. Crawford F, et al. Specificity and detection of insulin-reactive CD4 ${ }^{+} \mathrm{T}$ cells in type 1 diabetes in the nonobese diabetic (NOD) mouse. Proc Natl Acad Sci USA. 2011;108(40):16729-16734.

16. Wong FS, et al. Identification of an MHC class I-restricted autoantigen in type 1 diabetes by screening an organ-specific cDNA library. Nat Med. 1999;5(9):1026-1031.

17. Nakayama M, et al. Prime role for an insulin epitope in the development of type 1 diabetes in NOD mice. Nature. 2005;435(7039):220-223.

18. Martinez RJ, et al. Arthritogenic self-reactive $\mathrm{CD} 4^{+} \mathrm{T}$ cells acquire an $\mathrm{FR} 4^{\mathrm{hi}} \mathrm{CD} 73^{\mathrm{hi}}$ anergic state in the presence of Foxp3 $3^{+}$regulatory T cells. J Immunol. 2012;188(1):170-181.

19. Pauken KE, et al. Cutting edge: type 1 diabetes occurs despite robust anergy among endogenous insulin-specific CD4 T cells in NOD mice. J Immunol. 2013;191(10):4913-4917.

20. Thomas HE, et al. Interferon signalling in pancreatic beta cells. Front Biosci (Landmark Ed). 2009;14:644-656.

21. Aschenbrenner K, et al. Selection of Foxp $3^{+}$regulatory T cells specific for self antigen expressed and presented by Aire ${ }^{+}$medullary thymic epithelial cells. Nat Immunol. 2007;8(4):351-358.

22. Yang S, Fujikado N, Kolodin D, Benoist C, Mathis D. Immune tolerance. Regulatory T cells generated early in life play a distinct role in maintaining self-tolerance. Science. 2015;348(6234):589-594.

23. Gagnerault MC, Luan JJ, Lotton C, Lepault F. Pancreatic lymph nodes are required for priming of beta cell reactive T cells in NOD mice. J Exp Med. 2002;196(3):369-377.

24. Thornton AM, et al. Expression of Helios, an Ikaros transcription factor family member, differentiates thymic-derived from peripherally induced Foxp3 ${ }^{+}$T regulatory cells. J Immunol. 2010;184(7):3433-3441.

25. Ziegler AG, et al. Seroconversion to multiple islet autoantibodies and risk of progression to diabetes in children. JAMA. 2013;309(23):2473-2479.

26. Huseby ES, Sather B, Huseby PG, Goverman J. Age-dependent T cell tolerance and autoimmunity to myelin basic protein. Immunity. 2001;14(4):471-481.

27. He Q, et al. Thymic development of autoreactive T cells in NOD mice is regulated in an age-dependent manner. J Immunol. 2013;191(12):5858-5866.

28. Suri A, Walters JJ, Gross ML, Unanue ER. Natural peptides selected by diabetogenic DQ8 and murine I-A(g7) molecules show common sequence specificity. J Clin Invest. 2005;115(8):2268-2276.

29. Takiishi $\mathrm{T}$, et al. Reversal of autoimmune diabetes by restoration of antigen-specific tolerance using genetically modified Lactococcus lactis in mice. J Clin Invest. 2012;122(5):1717-1725.

30. Ma Y, et al. Oral administration of recombinant Lactococcus lactis expressing HSP65 and tandemly repeated P277 reduces the incidence of type I diabetes in non-obese diabetic mice. PLOS ONE. 2014;9(8):e105701.

31. Culina S, et al. Materno-fetal transfer of preproinsulin through the neonatal Fc receptor prevents autoimmune diabetes. Diabetes 2015;64(10):3532-3542.

32. Verdaguer J, Schmidt D, Amrani A, Anderson B, Averill N, Santamaria P. Spontaneous autoimmune diabetes in monoclonal T cell nonobese diabetic mice. J Exp Med. 1997;186(10):1663-1676.

33. Mollah ZU, et al. Granzyme B is dispensable in the development of diabetes in non-obese diabetic mice. PLoS ONE. 2012;7(7):e40357.

34. Nakayama M, et al. Priming and effector dependence on insulin B:9-23 peptide in NOD islet autoimmunity. J Clin Invest. 2007;117(7):1835-1843.

35. Moon JJ, et al. Tracking epitope-specific T cells. Nat Protoc. 2009;4(4):565-581.

36. Babaya N, Liu E, Miao D, Li M, Yu L, Eisenbarth GS. Murine high specificity/sensitivity competitive europium insulin autoantibody assay. Diabetes Technol Ther. 2009;11(4):227-233. 